\title{
An enhanced discrete Mindlin finite element model using a zigzag function
}

\author{
Lakhdar Sedira $^{\mathrm{a}, \mathrm{b} *}$, Rezak Ayad ${ }^{\mathrm{b}}$, Hamid Sabhi $^{\mathrm{b}}$, Mabrouk Hecini $^{\mathrm{a}}$ and Siham Sakami ${ }^{\mathrm{c}}$ \\ ${ }^{a}$ Mechanical Engineering Laboratory (LGM), University of Biskra, BP145, 07000 Biskra, Algeria; \\ ${ }^{b}$ Laboratory of Engineering and Material Sciences, University of Reims Champagne-Ardenne, ESIEC, \\ Esp. Rolland Garros, BP 1029, F-51686, Reims, France; ${ }^{c}$ Faculty of Sciences and Technology, Group \\ of Research in Civil and Geo-Engineering, BP 549, Av. Abdelkarim Elkhattabi, Guéliz, Marrakech,
} Morocco

\begin{abstract}
The present work deals with the formulation and the evaluation of a discrete finite element model for Reissner/Mindlin composite plates, including the introduction of zigzag form in order to improve plane and shear stress accuracy. The model is characterised by a piecewise linear variation of displacement, which allows to fulfil the stress continuity requirements. For this purpose, a new four-node quadrilateral enhanced finite element based on a quadratic displacement field is proposed. In the second version, it incorporates two additional zigzag terms and does not require shear correction. The element is validated across some known problems in the literature, highlighting the improvement of thickness stress distributions, by comparison with the initial model without zigzag function.

Le présent travail traite de la formulation et l'évaluation d'un model d'élément fini discret de Reissner/Mindlin pour les plaques composites, incluant l'introduction de la forme en zigzag en vue d'améliorer la précision de contraintes planes et de cisaillement transversal (CT). Le modèle est caractérisé par une variation linéaire par couche du déplacement, ce qui permet de satisfaire aux exigences de continuité des contraintes. En conséquence, un nouvel élément fini à quatre nœuds amélioré, avec un champ de déplacements quadratique, est proposé. Dans une seconde version, il comporte deux termes additionnels de zigzag et ne nécessite pas une correction du CT. L'élément est validé à travers quelques problèmes tests connus de la littérature, mettant en évidence notamment une amélioration des distributions de contraintes à travers l'épaisseur, par rapport au modèle initial sans la fonction zig-zag.
\end{abstract}

Keywords: finite element; displacement discrete model; multilayer plate; zigzag function

Mots-clés: élément fini; modèle déplacement discret; plaque multicouche; fonction zigzag

\section{Introduction}

In the last decades, important theories for modelling and analysing of composite and sandwich plates were developed. The aim is to reduce the $3 \mathrm{D}$ problems to $2 \mathrm{D}$ ones and to respect the conditions derived from the continuum mechanics. Despite the fact that these requirements are never simultaneously realised, some theories for finite elements application appear as an adequate model.

*Corresponding author. Email: 1.sedira@univ-biskra.dz 
Examining performances of some theories mentioned in specialised reviews and continuity requirement in revolution composite structures, where the mechanical, piezoelectric and thermal behaviours are coupled. Although it is well known that the first-order deformation theory alone is unable to represent reliable local effects in composite structures, scientists have always a tendency to preserve the global part of kinematics field in their improved formulation. That is why elements based on first-order theory (Reissner/Mindlin or Kirchhoff theories) are still in development and remain inescapable, as for the isotropic or multilayer structures. Good global responses are obtained by using Reissner-Mindlin or mixed Hellinger-Reissner models and by introducing additional techniques to eliminate defects created by several computational mechanisms. These techniques have greatest importance and are more justified for large-scale non-linear analysis. For this context, exhaustive references are cited in the literature and refer to the pioneers of works (Mindlin, 1951; Reissner, 1943).

In the early 1980s, much work has been devoted under the well-known discrete Kirchhoff formulation (integral sense). Thereafter, several finite elements have been developed successfully based on this concept (Batoz \& Tahar, 1982). Unfortunately, these elements were incapable of presenting thick plate behaviour. For this purpose, the Mindlin hypotheses become a necessity for the subsequent developments, citing elements formulated for isotropic plates, well-known, simple and robust (Ayad, Dhatt, \& Batoz, 1998; Katili, 1993; Lardeur, 1990). Although this formulation leads often to a constant shear strain element and a shear parameter, the technique remains convincing for use as support to any future improved formulation for multilayer composites.

In recent years, a great part of research has been focused on finite element formulation of models which provide accurate prediction of the transverse shear stresses. The adoption of the last approach constitutes one among our current interest items, where a new displacement variational model, called Displacement Discrete Mindlin (DDM), is developed (Ayad, 2002). The effect of transverse shear is taken into account. The obtained elements pass the patch tests. Also, they are immune to all numerical problems (locking, distortion, etc.) (Sakami, 2008). In order to apply this concept for composite structures, two local modified discrete Mindlin hypotheses have been introduced to the isotropic model. The first assumption is kinematic; it consists of introducing, as a contour integral form, a kinematics equation of transverse shear deformation. It allows the elimination of transverse shear locking without introducing bubble functions as well as recourse to the reduced integration, which is commonly known as assumed natural strain. The second is mechanical hypothesis. While the multilayered plate aspect is respected, both bending and transverse shear behavioural laws and two equilibrium equations are used. Its main advantage is the local elimination of mid-side rotation degrees of freedom (DOF), initially assumed to have a quadratic approximation.

In the second part of the present work, with the aim to overcome constant shear strain along the element, an improved technique is required. According to many reviews on materials approaches and formulation of multilayered plates, several scientists have concluded that the verification of the inter-laminar stress continuity necessarily requires a transverse strain discontinuity. This condition can be concretised by the piecewise linear or non-linear displacement, i.e. zigzag form. In fact, among the proposed models, three fundamental approaches can be found. They are derived from Lekhnitskii, Ambartsumian and Reissner works. More detailed classification is given in Carrera (2002). The first zigzag theory has been given by Lekhnitskii (1935) for beam frameworks. Ambartsumian (1969) added thereafter noteworthy contributions to the plate and shell theories. The Lekhnitskii method was rarely considered in the literature. The approach of Amartsumian-Whitny-Rath-Das (Ambartsumian, 1969; Rath \& Das, 1973; Whitney, 1969) is also a technique that takes into account zigzag form in distribution of displacements, it has the particularity to preserve the same number of unknown variables as that 
of First-order Shear Deformation Theory (FSDT), i.e. three displacements and two rotations (or shear strain). The last approach has been recently developed by Murakami (1986), it is based on the addition of a zigzag function. A brief description is given in a subsequent section.

Briefly, based on modified discrete Mindlin hypotheses, a four-node, bending/shear multilayer anisotropic plate element of quadrilateral shape is proposed. The element incorporates only three nodal DOF, namely transverse displacement and two quadratic rotations. In order to involve a piecewise linear displacement to present model, a second form based on Murakami zigzag approach, requires two additional displacement terms, is evaluated.

\section{Theoretical formulation of the DMQP plate model}

The new plate element, called Discrete Mindlin quadrilateral element for multilayer plate (DMQP), is an extension to the multilayer case of the discrete Kirchhoff-Mindlin quadrilateral isotropic model proposed by Katili (1993) that could be obtained by equivalent model of MiSP4 + (four-node quadrilateral element based on mixed shear projection model) (Ayad, 2002; Ayad \& Rigolot, 2002).

Initially, DMQP/ml element is based on eight-node quadrilateral element, baptised DMQP $\beta$, then again reduced to four nodes only. It preserves the quadratic kinematics fields in the final form, with three DOF per node $\left(w, \beta_{x}\right.$, and $\beta_{y}$ ): the transverse displacement along the $\mathrm{z}$-axis and two rotations of the normal to the mid-surface in two planes $x-z$ and $y-z$, respectively.

\subsection{Displacement field}

Description of displacement fields of the present model for shear-bending problem is devoted to its simplified version; Equation (1) which is based on first-order theory, the effect of the additional zigzag terms can be introduced into the DDM model with only in-plane displacement variables. However, their expressions will not be explicitly mentioned in this context, for more details on the theoretical zigzag model (Section 4) the author can refer to (Carrera, 1996, 2002; Murakami, 1986).

$$
\vec{u}_{\mathrm{q}}(x, y, z)=\left\{\begin{array}{c}
\vec{u}(x, y, z) \\
\vec{v}(x, y, z) \\
\vec{w}(x, y, z)
\end{array}\right\}=\left\{\begin{array}{c}
0 \\
0 \\
\vec{w}(x, y)
\end{array}\right\}+z\left\{\begin{array}{c}
\vec{\beta}_{x}(x, y) \\
\vec{\beta}_{y}(x, y) \\
0
\end{array}\right\}
$$

As the transverse displacement $w$ is assumed linear, we propose to approach the rotations $\beta_{x}$ and $\beta_{y}$ with an incomplete quadratic interpolation that will reveal increases of rotations $\Delta \beta_{s k}$ on all four elementary sides. $\Delta \beta_{s k}$ are the variables associated with the quadratic representation of $\beta_{x}$ and $\beta_{y}$.

They are given by the expression below:

$$
\begin{gathered}
w=\sum_{i=1}^{4} N_{i} w_{i} \\
\left\{\begin{array}{l}
\beta_{x} \\
\beta_{y}
\end{array}\right\}=\sum_{i=1}^{4} N_{i}\left\{\begin{array}{l}
\beta_{x i} \\
\beta_{y i}
\end{array}\right\}+\sum_{k=5}^{-8} P_{k}\left\{\begin{array}{l}
C_{k} \\
S_{k}
\end{array}\right\} \Delta \beta_{s k}
\end{gathered}
$$

$\beta_{x i}$ and $\beta_{y i}$ are the nodal rotations at the quadrilateral corners $1-4 . \Delta \beta_{x k}$ is the nodal increment of the tangential rotation at the mid-nodes 5-8. $N_{i}$ and $P_{k}$ are the interpolating shape functions prescribed, respectively, in terms of linear and quadratic independent functions (Table 1). 
Table 1. Shape functions used in DMQP.

\begin{tabular}{lc}
\hline$\left\{N_{i}\right\}$ & $\left\{P_{k}\right\}$ \\
\hline $4 N_{1}=(1-\xi)(1-\eta)$ & $2 P_{5}=\left(1-\xi^{2}\right)(1-\eta)$ \\
$4 N_{2}=(1+\xi)(1-\eta)$ & $2 P_{6}=(1+\xi)\left(1-\eta^{2}\right)$ \\
$4 N_{3}=(1+\xi)(1+\eta)$ & $2 P_{7}=\left(1-\xi^{2}\right)(1+\eta)$ \\
$4 N_{1}=(1-\xi)(1+\eta)$ & $2 P_{8}=(1-\xi)\left(1-\eta^{2}\right)$ \\
\hline
\end{tabular}

The coefficients $C_{k}$ and $S_{k}$ are the direction cosines of vector associating node $i-j$ of side $k$ (Figure 2).

\subsection{Strain field}

The strain field is obtained by substituting Equation (1) into the linear strain-displacement relation, which allows us, first, to write:

$$
\left\langle\varepsilon_{t}\right\rangle=\left\langle\left\langle\varepsilon_{\mathrm{s}}\right\rangle\left\langle\gamma_{\mathrm{s}}\right\rangle\right\rangle, \quad \varepsilon_{z z}=0
$$

$\left\langle\varepsilon_{S}\right\rangle$ and $\left\langle\gamma_{s}\right\rangle$ are, respectively, the bending and transverse shear strains, with:

$$
\left\langle\varepsilon_{\mathrm{s}}\right\rangle=\left\langle\begin{array}{lll}
\varepsilon_{x} & \varepsilon_{y} & \gamma_{x y}
\end{array}\right\rangle=z\langle\chi\rangle
$$

where, the bending curvature, is defined as:

$$
\langle\chi\rangle=\left\langle\begin{array}{lll}
\beta_{x, x} & \beta_{y, y} & \beta_{x, y}+\beta_{y, x}
\end{array}\right.
$$

Substitution of the nodal interpolation of rotations, Equation (2) into Equation (5), yields:

$$
\langle\chi\rangle=\left[B_{\mathrm{f}}\right]\left\{\begin{array}{c}
u_{n} \\
\Delta \beta_{n}
\end{array}\right\}=\left[B_{b \beta}\right]\left\{u_{n}\right\}+\left[B_{b \Delta \beta}\right]\left\{\Delta \beta_{n}\right\}
$$

where

$$
\left[B_{b \beta}\right]=\left[\begin{array}{ccccc}
0 & a_{i} & 0 & \cdots \\
0 & 0 & b_{i} & \cdots & i=1,2,3,4 \\
0 & b_{i} & a_{i} & \cdots &
\end{array}\right], \quad \begin{aligned}
& a_{i}=j_{11} N_{i, \xi}+j_{12} N_{i, \eta} \\
& b_{i}=j_{21} N_{i, \xi}+j_{22} N_{i, \eta}
\end{aligned}
$$

$[j]$ is the inverse Jacobian matrix, defined as:

$$
[j]=\frac{1}{\operatorname{det}[J]}\left[\begin{array}{ll}
y_{, \eta} & y, \xi \\
x, \eta & x, \xi
\end{array}\right], \quad[J]=\left[\begin{array}{ll}
x, \xi & y, \xi \\
x, \eta & y, \eta
\end{array}\right]
$$

and

$$
\left[B_{b \Delta \beta}\right]=\left[\begin{array}{cl}
C_{k}\left(j_{11} P_{k, \xi}+j_{12} P_{k, \eta}\right) & \cdots \\
S_{k}\left(j_{21} P_{k, \xi}+j_{22} P_{k, \eta}\right) & \cdots \\
C_{k}\left(j_{21} P_{k, \xi}+j_{22} P_{k, \eta}\right)+S_{k}\left(j_{11} P_{k, \xi}+j_{12} P_{k, \eta}\right) & \cdots
\end{array}\right]
$$




$$
\begin{aligned}
& \left\langle U_{n}\right\rangle=\left\langle w_{1} \beta_{x 1} \beta_{y 1} w_{2} \beta_{x 2} \beta_{y 2} w_{3} \beta_{x 3} \beta_{y 3} w_{4} \beta_{x 4} \beta_{y 4}\right\rangle \\
& \left\langle\Delta \beta_{n}\right\rangle=\left\langle\Delta \beta_{s 5} \Delta \beta_{s 6} \Delta \beta_{s 7} \Delta \beta_{s 8}\right\rangle
\end{aligned}
$$

The transverse shear strains can be written in the following form:

$$
\left\{\gamma_{\mathrm{s}}\right\}=\left\{\begin{array}{l}
\gamma_{x z} \\
\gamma_{y z}
\end{array}\right\}=\left\{\gamma_{0}\right\}
$$

where

$$
\left\langle\gamma_{0}\right\rangle=\left\langle w_{0, x}+\beta_{x} \quad w_{0, y}+\beta_{y}\right\rangle
$$

We define the Cartesian strain of transverse shear in function of isoparametric strain $\left\{\gamma_{\zeta}\right\}$. They are linearly interpolated, two by two, on each elementary side (Figure 1).

$$
\begin{gathered}
\left\{\gamma_{0}\right\}=[j]\left\{\begin{array}{l}
\gamma_{\xi z} \\
\gamma_{\eta z}
\end{array}\right\} \\
\left\{\begin{array}{l}
\gamma_{\xi z} \\
\gamma_{\eta z}
\end{array}\right\}=\left\{\begin{array}{l}
\frac{1}{2}(1-\eta) \gamma_{\xi \zeta 5}+\frac{1}{2}(1+\eta) \gamma_{\xi \zeta 7} \\
\frac{1}{2}(1-\xi) \gamma_{\eta \varsigma 8}+\frac{1}{2}(1+\xi) \gamma_{\eta \zeta 6}
\end{array}\right\}
\end{gathered}
$$

$\gamma_{\xi \zeta 5}, \gamma_{\eta \zeta 6}, \gamma_{\xi \zeta 7}$ and $\gamma_{\eta \zeta 8}$ are the isoparametric shear strains, at sides 5, 6, 7 and 8, respectively. They are related to edge tangential shear strains $\gamma_{s 5}, \gamma_{s 6}, \gamma_{s 7}$ and $\gamma_{s 8}$ as follows:

$$
\gamma_{\xi \xi k}=\operatorname{det}\left(J_{s k}\right) \gamma_{s k}
$$

The following expressions can be obtained by substituting the coordinates of each $k$ th midside in Equation (16);

$$
\gamma_{\xi \zeta 5}=\frac{L_{5}}{2} \gamma_{s 5}, \quad \gamma_{\xi \zeta 7}=-\frac{L_{7}}{2} \gamma_{s 7}, \quad \gamma_{\eta \zeta 6}=\frac{L_{6}}{2} \gamma_{s 6}, \quad \gamma_{\eta \zeta 8}=-\frac{L_{8}}{2} \gamma_{s 8}
$$

where $L_{k}$ is the length of side $k$.

Therefore, $\left\{\gamma_{0}\right\}$ will then be written in terms of $\left\{\gamma_{s k}\right\}$ as:

$$
\left\{\gamma_{0}\right\}=[N \gamma]\left\{\gamma_{s k}\right\}
$$

where

$$
\left\{N_{\gamma}\right\}=\frac{1}{4}\left[\begin{array}{llll}
L_{5} J_{11}(1-\eta) & L_{6} J_{12}(1+\xi) & -L_{7} J_{11}(1+\eta) & -L_{8} J_{12}(1-\xi) \\
L_{5} J_{21}(1-\eta) & L_{6} J_{22}(1+\xi) & -L_{7} J_{21}(1+\eta) & -L_{8} J_{22}(1-\xi)
\end{array}\right]
$$

and

$$
\left\{\gamma_{s k}\right\}^{\mathrm{T}}=\left\langle\begin{array}{llll}
\gamma_{s 5} & \gamma_{s 6} & \gamma_{s 7} & \gamma_{s 8}
\end{array}\right\rangle
$$

These terms, will be projected on the elementary DOF $\left\{U_{n}\right\}$ and $\left\{\Delta \beta_{s k}\right\}$, are defined by a contour integral along either side $k$. 


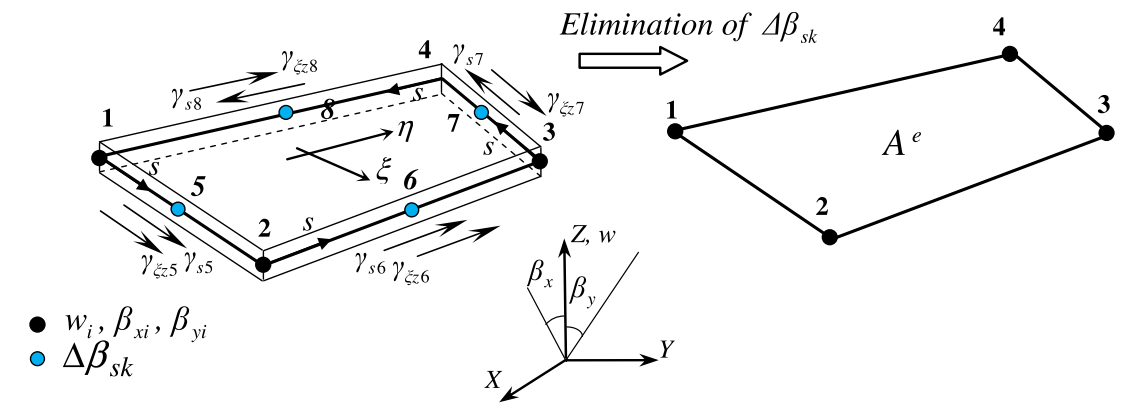
(a) Initial element: $D M Q P \beta$ (quadratic Rotations)

(b) Final element $D M Q P$

Figure 1. DMQP plate element.

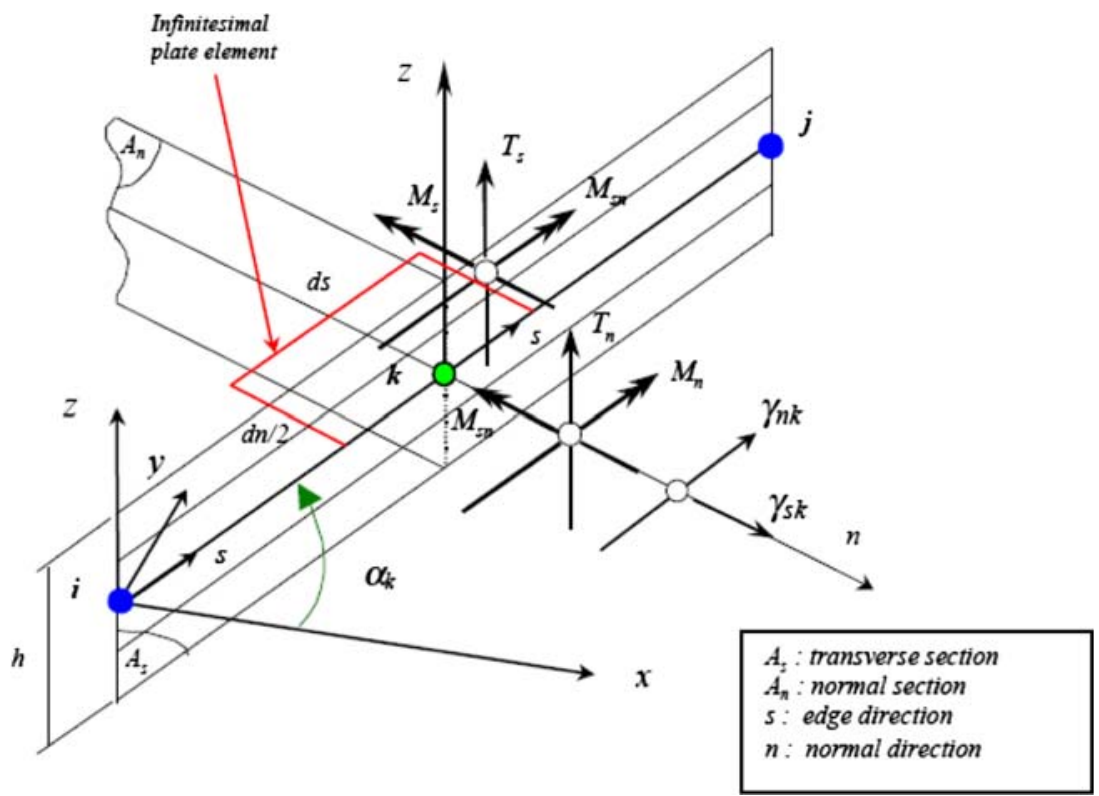

Figure 2. Modified Mindlin hypotheses on the side $k$ of a multilayer element.

$$
\int_{0}^{L_{k}}\left\{\gamma_{s k}-\tilde{\gamma}_{s k}\right\} d s=0 \quad \text { (Kinematic Hypothesis) }
$$

where

$$
\tilde{\gamma}_{s k}=\left(w, \beta_{\mathrm{s}}\right)_{k}
$$

and

$$
\begin{gathered}
\beta_{\mathrm{s}}=\left(1-\frac{s}{L_{k}}\right) \beta_{s i}+\frac{s}{L_{k}} \beta_{s j}+4 \frac{s}{L_{k}}\left(1-\frac{s}{L_{k}}\right) \Delta \beta_{s k} \\
\beta_{n}=\left(1-\frac{s}{L_{k}}\right) \beta_{n i}+\frac{s}{L_{k}} \beta_{n j}
\end{gathered}
$$


Introducing kinematic hypothesis (21) on the side $k$, which are projected on the element using above interpolations of $\beta_{s}$ and $\beta_{n}(23)$, lets us rewrite the edge tangential shear strains as follows:

$$
\gamma_{s k}=\frac{w_{j}-w_{i}}{L_{k}}+\frac{1}{2}\left(\beta_{s i}+\beta_{s j}\right)+\frac{2}{3} \Delta \beta_{s k}
$$

In order to eliminate the nodal variables of the mid-sides $\Delta \beta_{s k}$, we have opted for a heuristic method that equalises the shear force calculated by Hooke's law and their values obtained from equilibrium equations of moments, respecting the multilayered composite plate character. This technique is considered as a modified Mindlin hypotheses (Ayad, 2002; Ayad, Talbi, \& Ghomari, 2009; Sakami, 2008).

Considering tangential strain as function of shear force, we can write the following local modified expression.

$$
\int_{0}^{L_{k}}\left(\gamma_{s k}-\left\langle H_{c k 11}^{\mathrm{inv}} \quad H_{c k 12}^{\mathrm{inv}}\right\rangle\left\{\begin{array}{l}
T_{\mathrm{s}} \\
T_{n}
\end{array}\right\}\right) d s=0 \quad \text { (Mechanical hypothesis) }
$$

$H_{c k 11}^{\text {inv }}$ and $H_{c k 12}^{\text {inv }}$ are two shear behaviour terms of the inverse matrix $\left[H_{c k}\right]^{-1}$ for the $k$ th side: where

$$
\begin{gathered}
{\left[H_{c k}\right]=\left[P_{k}\right]\left[H_{\mathrm{c}}\right]\left[P_{k}\right]^{-1}} \\
{\left[P_{k}\right]=\left[P_{k}\right]^{-1}=\left[\begin{array}{cc}
C_{k} & S_{k} \\
S_{k} & -C_{k}
\end{array}\right], \quad\left\{\begin{array}{l}
C_{k}=\cos \theta_{k} \\
S_{k}=\sin \theta_{k}
\end{array}\right.}
\end{gathered}
$$

$\left[H_{c}\right]$ is the constitutive transverse shear of the entire multilayer and $\theta_{k}$ is the angle between $\overrightarrow{i j}$ vector, defined by $i$ and $j$ nodes of the $k$ side, and the $x$-axis.

$T_{s}$ and $T_{n}$ are the transverse shear forces, we use the equilibrium relations on middle surface of the side $k$ to calculate their values in terms of bending moments. They are written as follows:

$$
\begin{aligned}
& T_{\mathrm{s}}=M_{s, s}+M_{s n, n} \\
& T_{n}=M_{s n, s}+M_{n, n}
\end{aligned}
$$

where

$$
\{M\}=\left\{\begin{array}{l}
M_{\mathrm{s}} \\
M_{n} \\
M_{s n}
\end{array}\right\}=\left[H_{\mathrm{f}}\right]\{\chi\}
$$

The homogenised stiffness matrix is given as:

$$
\left[H_{\mathrm{f}}\right]=\int_{-h / 2}^{+h / 2} z^{2}[H(z)] d z
$$

$\{\chi\}$ is the bending curvatures defined as: 


$$
\{\chi\}=\left\{\begin{array}{c}
\chi_{\mathrm{s}} \\
\chi_{n} \\
\chi_{s n}
\end{array}\right\}=\left\{\begin{array}{c}
\beta_{s, s} \\
\beta_{n, n} \\
\beta_{s, n}+\beta_{n, s}
\end{array}\right\}
$$

We define the strains $\left\{\gamma_{s k}\right\}$ as function of second derivatives of $\beta_{\mathrm{s}}$ and $\beta_{n}$ through Equation (28) and two constitutive laws in bending and shear. We obtained:

$$
\gamma_{s k}=-\varphi_{k}^{\mathrm{ml}} \Delta \beta_{s k}
$$

$\varphi_{k}^{\mathrm{ml}}$ is the influence factors of transverse shear, defined at either side as:

$$
\varphi_{k}^{\mathrm{ml}}=8\left(H_{f 11} \frac{H_{c k 11}^{\mathrm{inv}}}{L_{k}^{2}}+H_{f 13} \frac{H_{c k 12}^{i n v}}{L_{k}^{2}}\right)
$$

The combination of the two Equations (24) and (32) gives us:

$$
\Delta \beta_{s k}=\frac{3}{2+3 \varphi_{k}^{\mathrm{ml}}}\left[\frac{w_{i}-w_{j}}{L_{k}}-\frac{1}{2}\left(\beta_{1 s i}+\beta_{1 s j}\right)\right]
$$

The substitution of the last equation on either elementary $k$ side permits to write the nodal displacements of the mid-sides as a function of those of corners.

$$
\left\{\Delta \beta_{n}\right\}=\left[A_{n}\right]\left[A_{G 0}\right]\left\{u_{n}\right\}
$$

Finally, the Cartesian transverse shear is obtained by replacing both Equations (32) and (35) in (18)

$$
\left\{\gamma_{0}\right\}=\left[N_{\gamma}\right]\left[A_{\varphi}\right]\left[A_{n}\right]\left[A_{G 0}\right]\left\{u_{n}\right\}
$$

In terms of influence factors of transverse shear, the diagonal square matrices $\left[A_{\varphi}\right]$ and $\left[A_{n}\right]$ are read as follows:

$$
\begin{aligned}
& {\left[A_{\varphi}\right]=\left[\begin{array}{cccc}
-\varphi_{5}^{\mathrm{ml}} & 0 & 0 & 0 \\
0 & -\varphi_{6}^{\mathrm{ml}} & 0 & 0 \\
0 & 0 & -\varphi_{7}^{\mathrm{ml}} & 0 \\
0 & 0 & 0 & -\varphi_{8}^{\mathrm{ml}}
\end{array}\right]} \\
& {\left[A_{n}\right]=\left[\begin{array}{cccc}
\frac{3}{2+3 \varphi_{5}^{\mathrm{ml}}} & 0 & 0 & 0 \\
0 & \frac{3}{2+3 \varphi_{6}^{\mathrm{ml}}} & 0 & 0 \\
0 & 0 & \frac{3}{2+3 \varphi_{7}^{\mathrm{ml}}} & 0 \\
0 & 0 & 0 & \frac{3}{2+3 \varphi_{8}^{\mathrm{ml}}}
\end{array}\right]}
\end{aligned}
$$

where $\left[A_{G 0}\right]$ is the matrix of dimension $4 \times 12$, defined by length and direction cosines of vector element sides. 


$$
\left[A_{G 0}\right]=\frac{1}{2}\left[\begin{array}{cccccc}
2 / L_{5} & -C_{5} & -S_{5} & -2 / L_{5} & -C_{5} & -S_{5} \\
0 & 0 & 0 & 2 / L_{6} & -C_{6} & -S_{6} \\
0 & 0 & 0 & 0 & 0 & 0 \\
-2 / L_{8} & -C_{8} & -S_{8} & 0 & 0 & 0 \\
0 & 0 & 0 & 0 & 0 & 0 \\
-2 / L_{6} & -C_{6} & S_{6} & 0 & 0 & 0 \\
2 / L_{7} & -C_{7} & -S_{7} & -2 / L_{7} & -C_{7} & -S_{7} \\
0 & 0 & 0 & 2 / L_{8} & -C_{8} & -S_{8}
\end{array}\right]
$$

\section{Variational displacement formulation}

The expression of the principle of virtual work is defined by its generalised form as follows:

$$
W=\sum_{\text {elts }}\left(W_{\text {int }}^{\mathrm{e}}-W_{\mathrm{ext}}^{\mathrm{e}}\right)=0 ; \quad \forall w^{*}, \beta_{x}^{*}, \beta_{y}^{*}
$$

with $w^{*}=\beta_{x}^{*}=\beta_{y}^{*}=0 ; w=\bar{w}, \beta_{x}=\bar{\beta}_{x}, \beta_{y}=\bar{\beta}_{y}$ on $S_{\mathrm{u}}$.

Where $W_{\text {ext }}$ is the virtual work done by external forces and $W_{\text {int }}$ is the elementary internal work, which can be written as

$$
W_{\text {int }}^{\mathrm{e}}=W_{\mathrm{f}}^{\mathrm{e}}+W_{\mathrm{c}}^{\mathrm{e}}=\int_{V^{\mathrm{e}}}\left(<\varepsilon_{\mathrm{s}}^{*}>\left\{\sigma_{\mathrm{s}}\right\}+<\gamma_{\mathrm{s}}^{*}>\left\{\tau_{\mathrm{s}}\right\}\right) d V
$$

Using the constitutive laws of stress-strain, taking into account the multilayered aspect and the stratification technique, the explicit integration of the first term of the integral [39] is given as:

$$
W_{\mathrm{f}}^{\mathrm{e}}=\int_{A^{\mathrm{e}}}\left(\left\langle\chi^{*}\right\rangle\left[H_{\mathrm{f}}\right]\{\chi\}\right) d A
$$

where $A_{\mathrm{e}}$ is the elementary surface.

Under nodal form

$$
W_{\mathrm{f}}^{\mathrm{e}}=\left\langle u_{n}^{*}\right\rangle\left[k_{\mathrm{f}}^{\mathrm{e}}\right]\left\{u_{n}\right\}
$$

The element stiffness matrix regarding the bending part is written as:

$$
\left[k_{\mathrm{f}}^{\mathrm{e}}\right]=\int_{A^{\mathrm{e}}}\left(\left[B_{\mathrm{f}}\right]^{\mathrm{T}}\left[H_{\mathrm{f}}\right]\left[B_{\mathrm{f}}\right]\right) d A
$$

where

$$
\left[B_{\mathrm{f}}\right]=\left[B_{1 b \beta}\right]+\left[B_{1 b \Delta \beta}\right]\left[A_{n}\right]\left[A_{G 0}\right]
$$

Following the same procedure, the work done by internal shear force can be written as follows:

$$
W_{\mathrm{c}}^{\mathrm{e}}=\int_{A^{\mathrm{e}}}\left(\left\langle\gamma_{0}^{*}\right\rangle\left[H_{\mathrm{c}}\right]\left\{\gamma_{0}\right\}\right) d A
$$


The shear stiffness matrix can be obtained from the following form:

$$
W_{\mathrm{c}}^{\mathrm{e}}=\left\langle u_{n}^{*}\right\rangle\left[k_{\mathrm{c}}^{\mathrm{e}}\right]\left\{u_{n}\right\}
$$

where

$$
\left[k_{\mathrm{c}}^{\mathrm{e}}\right]=\int_{A^{\mathrm{e}}}\left[B_{c 0}\right]^{\mathrm{T}}\left[H_{\mathrm{c}}\right]\left[B_{c 0}\right] d A
$$

and

$$
\left[B_{c 0}\right]=\left[N_{\gamma}\right]\left[A_{\varphi}\right]\left[A_{n}\right]\left[A_{G 0}\right]
$$

The constitutive matrix is given as:

$$
\left[H_{\mathrm{c}}\right]=\int_{-h / 2}^{+h / 2}\left[H_{\tau}(z)\right] d z
$$

Finally, both elementary stiffness matrices $\left[k_{\mathrm{f}}^{\mathrm{e}}\right]$ and $\left[k_{\mathrm{c}}^{\mathrm{e}}\right]$ are obtained by an exact numerical integration scheme, i.e. $2 \times 2$ Gauss points. The homogeneous constitutive matrices are computed analytically through the direction $z$.

\section{General aspects of the zigzag function}

In the context of Reissner multilayer theory applications, Murakami has introduced for the first time, a function through the coordinate thickness, which is able to imitate the zigzag form (Carrera, 1996, 2002; Murakami, 1986). Such function, called "Murakami zigzag function," is defined as follows:

$$
M(z)=(-1)^{1} \zeta_{1}
$$

As $z$ is the coordinate thickness of the whole multilayered plate, $\zeta_{k}=2 z_{1} / h_{1}$ is an adimensional coordinate of the layer 1 , of thickness $h_{1}$. The shape function is illustrated in Figure 3. It has local properties of zero at the middle plane of each layer, (1) and $(-1)$ at the top and bottom interfaces, respectively. The theoretical strength lies in systematic fulfilment of inter-laminar continuity condition. Their terms can be easily inserted as much that in in-plane displacement field that in out-of-plane. This constitutes a further advantage compared to other zigzag theories: of Liknitskii as well as Ambartsumian (Carrera, 2002). We should note its wide applicability to all classical theories of multilayered plates (equivalent single layer) (Classical Laminate Theory, FSDT and High Shear Deformation Theory [HSDT]).

The application of the Murakami's zigzag formulation on DDM model can be summarised in the following form:

$$
\left\{\begin{array}{c}
\vec{u}(x, y, z) \\
\vec{v}(x, y, z) \\
\vec{w}(x, y, z)
\end{array}\right\}=\left\{\begin{array}{c}
0 \\
0 \\
\vec{w}(x, y)
\end{array}\right\}+z\left\{\begin{array}{c}
\vec{\beta}_{x}(x, y) \\
\vec{\beta}_{y}(x, y) \\
0
\end{array}\right\}+M(z)\left\{\begin{array}{c}
u_{1}^{\mathrm{M}}(x, y) \\
u_{2}^{\mathrm{M}}(x, y) \\
0
\end{array}\right\}
$$




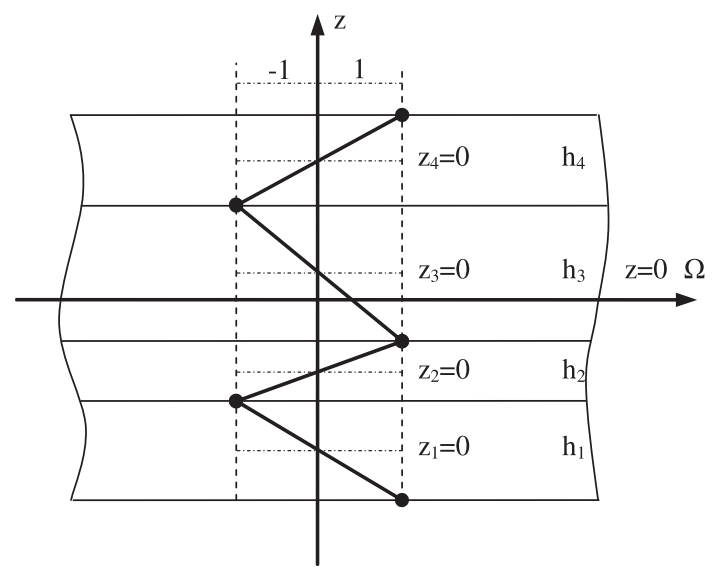

Figure 3. Geometrical representation of Murakami zigzag function (four-layer structure).

where the subscripts (1) and (2) indicate the vector displacement components in two directions of the plate coordinate system.

$u_{1}^{\mathrm{M}}$ and $u_{2}^{\mathrm{M}}$ are the effective amplitudes of the zigzag effect, having the sense of displacements. In fact, these terms have an intrinsic description of Equivalent single-layer model. This reality does not constitute a limitation of Murakami Zig-Zag Function, since it has the same description as in other multilayered theories of Ambartsumian and Liknitskii.

It should be also noted that the improved model of first-order shear deformation theory (with the Murakami zigzag function) has two or three additional DOF more than the classical model (FSDT).

\section{Numerical results and discussion}

Four numerical examples of multilayer composites and sandwich plates are solved. We proceed to compare obtained results with the first and higher order results. Moreover, hybridmixed variational approach is mentioned to carry out a correlation with our previous works regarding a Mindlin multilayered hybrid-mixed quadrilateral element (Tafla, Ayad, \& Sedira, 2010).

The boundary conditions are the same in all cases, the restrained DOF appropriate to simply supported conditions are given as follows:

$w=\beta_{x}=u_{1}^{\mathrm{M}}=0$, boundary line parallel to $x$-axis

$w=\beta_{y}=u_{2}^{\mathrm{M}}=0$, boundary line parallel to $y$-axis

\subsection{Example 1}

A cross-ply (0/90/0) rectangular laminate is considered, simply supported at the four edges using $6 \times 6$ mesh size of the quarter plate only (Figure 4). The plate is subjected to doubly sinusoidal load with intensity $q=q_{0} \sin (\pi x / a) \sin (\pi y / b)$. The three layers have equal thickness $h_{1}=h / 3$ and identical mechanical properties $\left(E_{x} / E_{y}=25, G_{x y}=G_{x z}=0.5 E_{y}, G_{y z}=.2 E_{y}\right.$ and $v=0.25)$. The analysis is carried out by taking two geometric aspects $b / a=1$ and 3 and different thickness ratio $a / h=4,10,50$ and 100 , where $h$ is the thickness of the plate. 

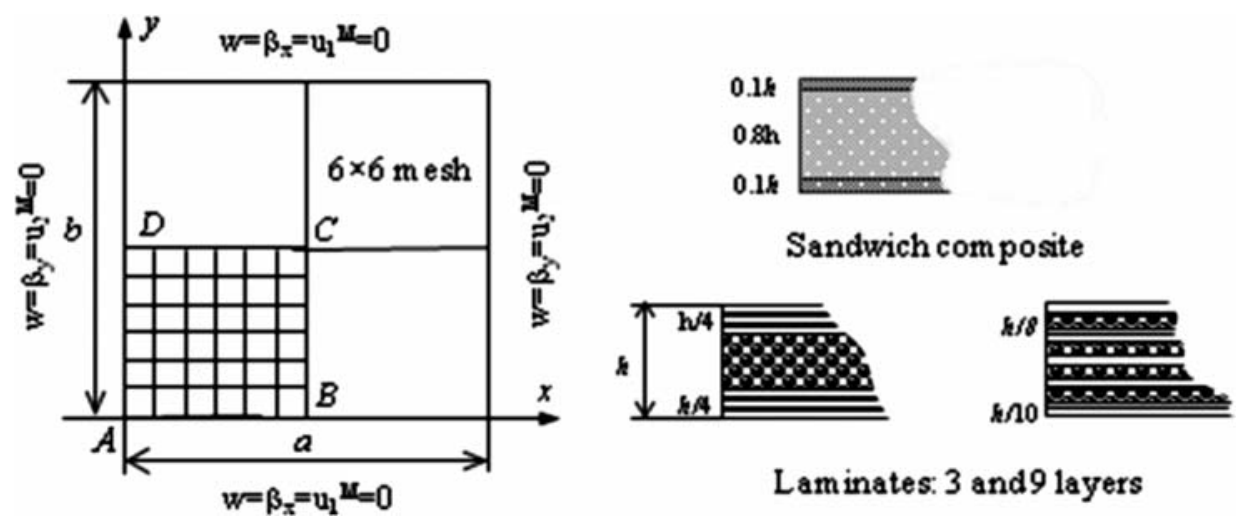

Laminates: 3 and 9 layers

Figure 4. Geometrical properties of sandwich and multilayered plates.

The results are compared with those of Di-Sciuva (1992) labelled RFSDT (Refined First-order Shear Deformation Theory) and HSDT. Few results for specific thickness ratio are furnished by Carrera and Demasi (2002) for the nine-node quadrilateral element with zigzag function, used by the author to evaluate the use of zigzag function in first-order shear deformation theory. Other elements and theoretical results are also used and listed as follows:

- DSQ: Discrete Shear Quadrilateral element (Lardeur, 1990).

- E.O.: Engblom \& Ochoa element, based on higher-order displacement theory. It is an eight nodes quadrilateral element with 10 DOFs (Engblom \& Ochoa, 1985).

- ED1, EDZ1: nine-node quadrilateral elements belong to equivalent single-layer model, based on first-order shear deformation theory and displacement formulation, the second one is formulated with zigzag function (Carrera \& Demasi, 2002).

- FSDTZZ: First Shear Deformation Theory with Zig-Zag, based on nonlinear fileds using a modified mixed variational formulation (Fares \& Elmarghany, 2008).

- PRHSDT: a four-node rectangular element based on Present Refined Higher-order Shear Deformation Theory (Topdar, Sheikh, \& Dhang, 2003).

- HSDT: higher order shear deformation theory (Reddy, 1984).

Deflection at the laminate centre and stresses are evaluated and written in terms of the following normalised quantities and locations:

$$
\begin{aligned}
w_{C} & =w\left(100 E_{Y} w h^{3} / q_{0} a^{4}\right), & \sigma_{x y A} & =\sigma_{x y}(0,0, h / 2) h^{2} q_{0} a^{2}, \\
\sigma_{x C} & =\sigma_{x}(a / 2, b / 2, h / 2) h^{2} / q_{0} a^{2}, & \tau_{x z D} & =\tau_{x z}(0, b / 2,0) h / q_{0} a, \\
\sigma_{y C} & =\sigma_{y}(a / 2, b / 2, h / 6) h^{2} q_{0} / a^{2}, & \tau_{y z B} & =\tau_{y z}(a / 2,0,0) h / q_{0} a
\end{aligned}
$$

The values of the transverse displacement, in-plane and transverse stresses for both shapes of the plate are shown in Tables 2 and 3. The accuracy of normal in-plane stress $\sigma_{x C}$ obtained by different first-order elements (DMQP/ml, DSQ) appears affected for the thick plate $(a /$ $h=4$ ). Whereas this is not the case for those obtained by DMQPz, where they appear more accurate. Both DDM models show a good performance regarding the in-plane stresses results $\left(\sigma_{y C}\right.$ and $\left.\tau_{x y A}\right)$ and central deflection, especially for thickness ratio $a / h=50$ and 100 (Table 3). 
Table 2. Deflection and stresses in square $(0 / 90 / 0)$ laminate plate under sinusoidal load, $(b / a=1)$.

\begin{tabular}{llllllll}
\hline$a / h$ & Models & $\bar{\sigma}_{x C}$ & $\bar{\sigma}_{y C}$ & $\bar{\tau}_{x y A}$ & $\bar{\tau}_{x z D}$ & $\bar{\tau}_{y z B}$ & $\bar{w}_{C}$ \\
\hline 4 & DMQP/ml & $\mp .392$ & $\mp .613$ & \pm .0439 & .305 & .218 & 2.238 \\
& DMQPz & $\mp .687$ & $\mp .524$ & \pm .0480 & .247 & .206 & 1.972 \\
& E.O. & $\mp .391$ & $\mp .572$ & \pm .0448 & .308 & .251 & - \\
& HSDT & $\mp .734$ & $\mp .503$ & \pm .050 & .286 & .209 & 1.922 \\
& RFSDT & $\mp .620$ & $\mp .661$ & \pm .045 & .262 & .254 & 1.891 \\
& Elasticity & -.755 & -.556 & .0505 & .256 & .217 & - \\
& & .801 & .534 & -.0511 & & & \\
10 & DMQP/ml & $\mp .5026$ & $\mp .293$ & \pm .0270 & .369 & .1115 & .7709 \\
& DMQPz & $\mp .5745$ & $\mp .280$ & \pm .0281 & .354 & .1154 & .7414 \\
& ED1 & -.5096 & -.2376 & .0055 & .1538 & .1117 & .7417 \\
& & .5113 & .2382 & -.0055 & & & \\
& EDZ1 & -.5625 & -.2757 & .0076 & .3777 & .1408 & .7417 \\
& & .5106 & .2762 & -.0076 & & & \\
& E.O. & $\mp .500$ & $\mp .279$ & \pm .0280 & .369 & .130 & - \\
& HSDT & $\mp .568$ & $\mp .269$ & .028 & .369 & .117 & .713 \\
& RFSDT & $\mp .549$ & $\mp .289$ & .027 & .364 & .123 & .723 \\
& Elasticity & -.5900 & -.288 & .0289 & .357 & .1228 & .7530 \\
& & .5900 & .285 & -.0289 & & & \\
50 & DMQP/ml & $\mp .540$ & $\mp .184$ & \pm .0213 & .3879 & .0829 & .4453 \\
& DMQPz & $\mp .5432$ & $\mp .183$ & \pm .0214 & .390 & .0878 & .4443 \\
& E.O. & $\mp .541$ & $\mp .164$ & \pm .233 & .392 & .0843 & - \\
& Elasticity & $\mp .541$ & $\mp .185$ & \pm .0234 & .393 & .0842 & - \\
100 & DMQP/ml & $\mp .541$ & $\mp .180$ & \pm .0211 & .3837 & .1039 & .4346 \\
& DMQPz & $\mp .542$ & $\mp .180$ & \pm .0212 & .389 & .107 & .4344 \\
& E.O. & $\mp .542$ & $\mp .167$ & \pm .0224 & .393 & .0827 & - \\
& Elasticity & $\mp .539$ & $\mp .181$ & \pm .0213 & .395 & .0828 & - \\
\hline
\end{tabular}

For a thick plate $(a / h=4)$, the DMQPz element shows a more significant improvement than DMQP model, remains comparable to that founded by refined high-order model. In comparison with piecewise approach, results obtained by the present zigzag model, for the aspect ratio $b / a=3$ (Table 3), appear quite similar to refined non-linear zigzag shear deformation theory (FSDTZZ), developed by Fares and Elmarghany (2008). On the whole, obtained values of transverse shear stresses remain satisfactory, even better than DSQ, E.O. and HSDT.

For a moderate thick multilayer square plate $(a / h=4)$, the in-plane displacement $u$ at point $\mathrm{B}$ and the stress $\sigma_{x x}$ in the centre of the plate are plotted (Figure 5). The curves are illustrated for two versions of DMQP elements. For the first case, a piecewise linear displacement is shown with a change of sign of displacement slope between layers. This curve is obtained by the addition of two functions: the linear standard term due to $\beta_{y}$ and the zigzag term related to the coefficient $(-1)^{1}$ and the additional degree of freedom, which is not equal to zero on this location.

The same effect was found for the stress $\sigma_{x x}$ curve, where the slope of the in-plane strain is slightly decreased, which results in greater stress values on the layer interfaces. In comparison with exact and FSDT results, (Pagano, 1970; Whitney, 1969), the obtained curves are quasi-identical to those given as benchmark (Pagano, 1970). In this example, hence the zigzag effect appears clearly in in-plane displacement and stress.

On the other hand, regarding the shear stress curves, the alternate sign of the additional displacement coefficient used thereafter in the equilibrium equation integral leads also to a slight curvature in $\tau_{x z}$ curve (Figure 6), where two inflexion points appeared in layer interfaces. 


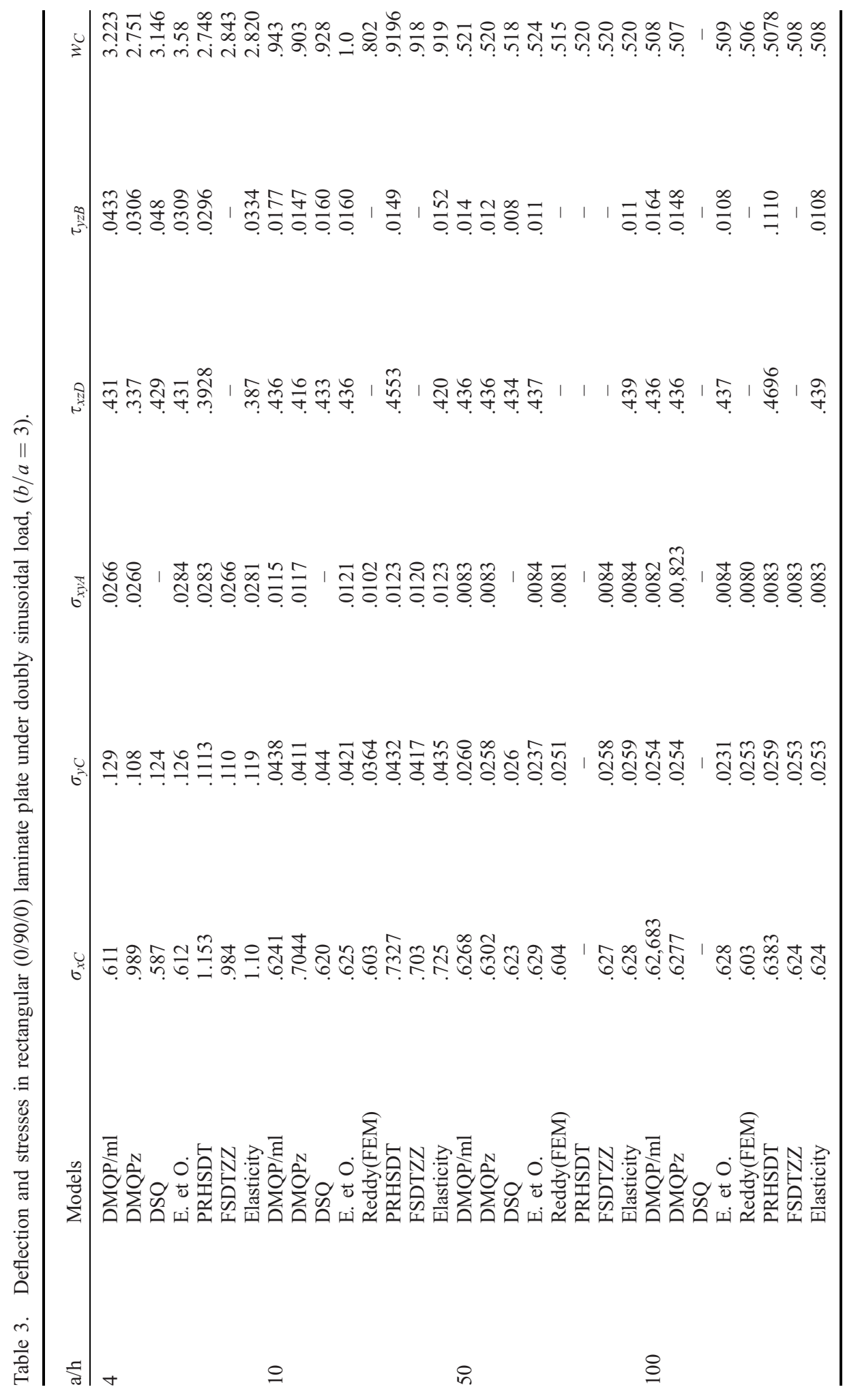



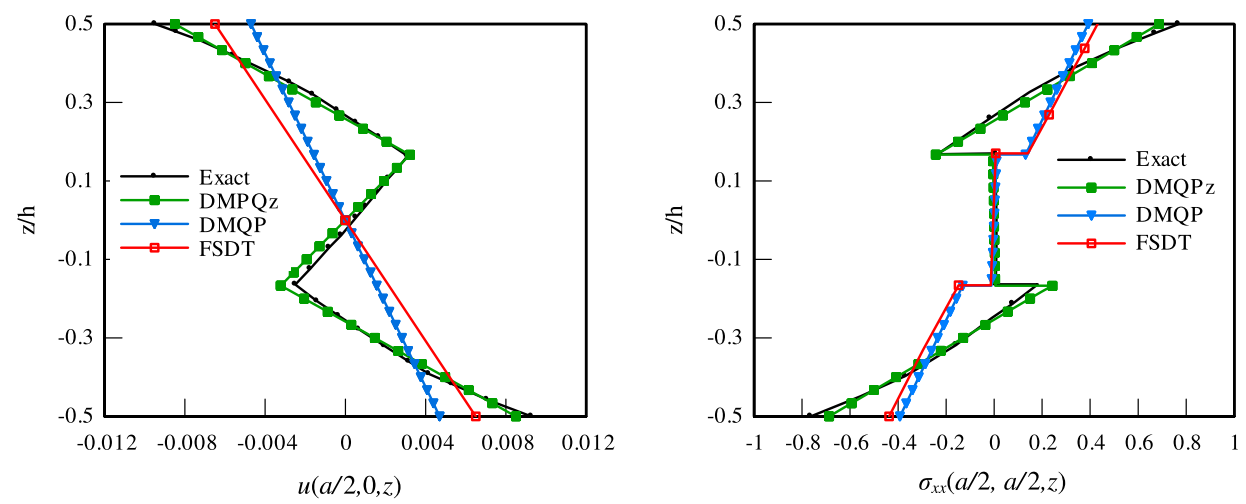

Figure 5. In-plane displacement and stress curves along the multilayer plate thickness, $a / h=4$.
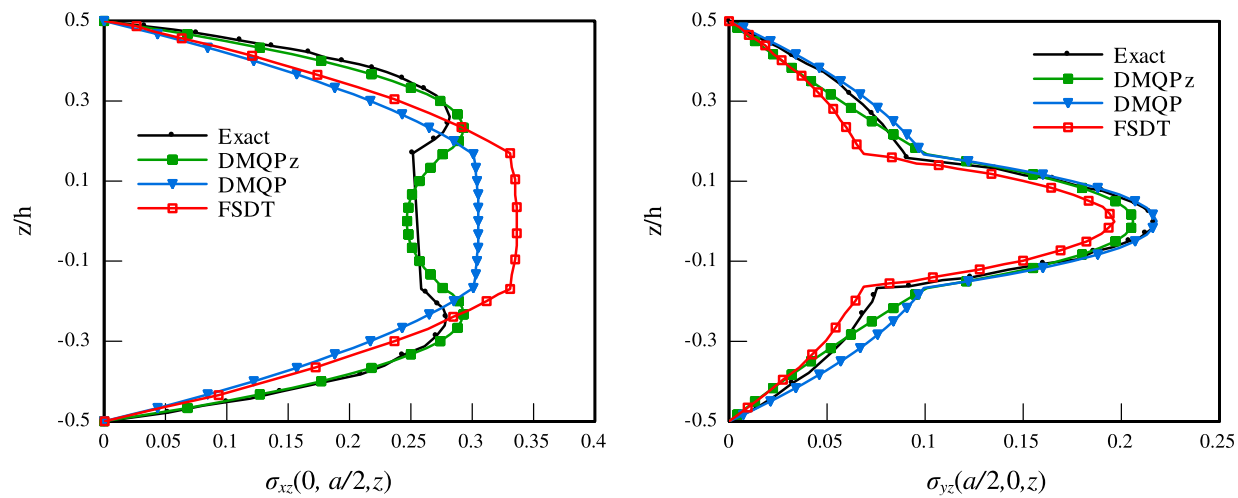

Figure 6. Distribution shear stresses along the multilayer plate thickness, $a / h=4$.

Usually, this could not have occurred in first-order shear deformation models. Both of the shear stress curves are quite close to 3D (Figure 6). So, the contribution of zigzag form introduced in DMQP model is more confirmed here.

\subsection{Example 2}

A laminated composite plate with a nine-layer symmetric cross-ply $[0 / 90 / 0 / 90 / 0]_{\text {sym }}$ made of unidirectional fibrous composite material is considered. The geometry, loading and material properties are assumed to be the same as those in example 1, except that the total thicknesses of layers $0^{\circ}$ and $90^{\circ}$ are equal, and layers of the same orientation have all the same thickness. Due to symmetry, only the quarter of plate is modelled using a $6 \times 6$ mesh. Transverse displacement and stresses $w$ at point C (Figure 4), in-plane stresses at points A and C and shear stresses $\tau_{x z}$ and $\tau_{y z}$ at points $\mathrm{D}$ and $\mathrm{B}$, respectively, are computed using the following adimensional form:

$$
w_{C}=w(a / 2, a / 2,0) \frac{\pi^{4} Q}{12 S^{4} h q_{0}}, \quad \sigma_{x C}=\sigma_{x}(a / 2, a / 2, h / 2) \frac{1}{q_{0} S^{2}}
$$




$$
\begin{gathered}
\sigma_{y C}=\sigma_{y}(a / 2, a / 2,2 h / 5) \frac{1}{q_{0} S^{2}}, \quad \sigma_{x y D}=\sigma_{x y}(0,0, h / 2) \frac{1}{q_{0} S^{2}} \\
\tau_{x z D}=\tau_{x z}(0, a / 2,0) \frac{1}{q_{0} S}, \quad \tau_{y z B}=\tau_{y z}(a / 2,0,0) \frac{1}{q_{0} S}
\end{gathered}
$$

with $Q=4 G_{12}+\frac{E_{1}+E_{2}\left(1+2 v_{23}\right)}{\left(1+v_{12} v_{21}\right)}, S=\frac{a}{h}$

The deflection obtained at the plate centre $(\mathrm{a} / 2, \mathrm{a} / 2,0)$ is accurately presented by the $\mathrm{DMQP} / \mathrm{ml}$ element, a good performance similar to the mixed model and better than the DSQ element. However, deflection in thick plate $(a / h=4$ and 10$)$ obtained by zigzag model is underestimated, but stresses are well presented nevertheless (Table 4).

\subsection{Example 3}

Aimed at evaluating the effect of heterogeneous anisotropic properties along the thickness on accuracy prediction of the present element, a square sandwich plate $(\mathrm{f} / \mathrm{c} / \mathrm{f})$ with thickness ratio $a / h=4$ and 10 was analysed. The plate is simply supported and subjected to a doubly sinusoidal load; it has a total thickness of $h$ where the thickness of the core is $0.8 h$ and that of each ply in the top and the bottom face sheets is $0.1 \mathrm{~h}$. Only the quarter of the plate is meshed $(\mathrm{DMQP} / \mathrm{ml} 8 \times 8$ and $19 \times 19$, DMQPz: $6 \times 6$ elements) (Figure 4). Mechanical properties are given as follows:

Face: $E_{x} / E_{y}=25, G_{x y}=G_{x z}=.5 E_{y}, G_{y z}=0.2 E_{y}, v=.25$

Core: $E_{x}=E_{y}=.04, G_{x z}=G_{y z}=.06, G_{x y}=.016, v=.25$

A reference solution from 3D elasticity is given for the first time by Pagano (1970). In addition, the comparison is carried out with results obtained by Topdar et al. (2003). With finite

Table 4. Deflection and maximum stresses in nine-layer square plate.

\begin{tabular}{llllllll}
\hline$a / h$ & Models & $\sigma_{x C}$ & $\sigma_{y C}$ & $\sigma_{x y A}$ & $\tau_{x z D}$ & $\tau_{y z B}$ & $w_{C}$ \\
\hline 4 & DMQP/ml & .475 & .511 & .0217 & .225 & .255 & 4.1872 \\
& DMQPz & .518 & .585 & .0224 & .218 & .264 & 3.6217 \\
& DSQ & .491 & .487 & - & .235 & .243 & 4.235 \\
& MiSP4/ml & .455 & .536 & - & .22 & .265 & 4.133 \\
& Elasticity & .684 & .628 & .0328 & .223 & .223 & 4.079 \\
10 & DMQP/ml & .5142 & .4646 & .0213 & .241 & .234 & 1.5171 \\
& DMQPz & .518 & .481 & .0215 & .240 & .235 & 1.4353 \\
& DSQ & .519 & .455 & - & .246 & .228 & 1.516 \\
& MiSP4/ml & .505 & .476 & - & .242 & .237 & 1.512 \\
& Elasticity & .551 & .477 & .0233 & .247 & .226 & 1.512 \\
50 & DMQP/ml & .541 & .432 & .0211 & .249 & .230 & 1.021 \\
& DMQPz & .541 & .434 & .0211 & .247 & .226 & 1.0175 \\
& DSQ & .538 & .432 & - & .253 & .216 & 1.015 \\
& MiSP4/ml & .54 & .435 & - & .256 & .218 & 1.019 \\
& Elasticity & .539 & .433 & .0214 & .258 & .219 & 1.021 \\
100 & DMQP/ml & .542 & .433 & .0210 & .244 & .250 & 1.0049 \\
& DMQPz & .542 & .433 & .0210 & .237 & .242 & 1.0042 \\
& Elasticity & .539 & .431 & .0213 & .259 & .219 & 1.005 \\
\hline
\end{tabular}


Table 5. Deflection and shear stresses in simply supported square sandwich $(\mathrm{f} / \mathrm{c} / \mathrm{f})$ plate, under sinusoidal load.

\begin{tabular}{|c|c|c|c|c|c|c|c|}
\hline & $\begin{array}{l}\mathrm{DMQP} / \mathrm{ml} \\
(8 \times 8)\end{array}$ & $\begin{array}{l}\mathrm{DMQP} / \mathrm{ml} \\
(19 \times 19)\end{array}$ & $\begin{array}{l}\text { DMQPz } \\
(6 \times 6)\end{array}$ & $\begin{array}{l}\text { HOZZT } \\
(8 \times 8) \\
\end{array}$ & $\begin{array}{l}\text { PRHSDT } \\
(8 \times 8) \\
\end{array}$ & $\begin{array}{l}\text { PFSDT } \\
(8 \times 8)\end{array}$ & Pagano \\
\hline \multicolumn{8}{|c|}{$a / h=4$} \\
\hline$w$ & 7.890 & 7.8656 & 7.6672 & 7.655 & 7.6042 & 4.7602 & 7.5962 \\
\hline$\tau_{x z}^{1}$ & .2525 & .255 & .2346 & .250 & .2592 & .1259 & .239 \\
\hline$\tau_{x z}^{2 z}$ & .2497 & .2525 & .2412 & - & .2136 & 1.0494 & - \\
\hline & .2497 & .2525 & - & - & .2136 & .1259 & - \\
\hline$\tau_{y z}^{1}$ & .1172 & $.11,775$ & .1057 & .1151 & - & - & .1072 \\
\hline$\tau_{x y}^{1}$ & .1269 & .1275 & .1459 & .1462 & - & - & .1484 \\
\hline \multicolumn{8}{|c|}{$a / h=10$} \\
\hline$w$ & 2.22 & 2.217 & 2.2083 & 2.2003 & 2.199 & 1.5601 & 2.5 \\
\hline$\tau_{x z}^{1}$ & .309 & .311 & .2972 & .3146 & .3256 & .1409 & .300 \\
\hline$\tau_{x z}^{2 z}$ & .307 & .310 & .2976 & - & .2683 & 1.1745 & - \\
\hline & .307 & .310 & - & - & .2683 & .1409 & - \\
\hline$\tau_{y z}^{1}$ & .0565 & .056 & .0521 & .056 & - & - & .0527 \\
\hline$\tau_{x y}^{1}$ & .0675 & .067 & .0708 & .070 & - & - & .0717 \\
\hline
\end{tabular}

element series: PFSDT (Present First-order Shear Deformation Theory), PRHSDT and the last one called HOZZT (Higher Order Zig-Zag Theory), it has the same order as the previous element and fulfils the zigzag form (based on cubic in-plane displacement fields, nine node and $11 \mathrm{DOFs}$ /node).

The transverse displacement and shear stresses at different points are presented in Table 5, with the next normalised. The proposed improved element provides results in very good agreement with the $3 \mathrm{D}$ elasticity. It shows competitive performance compared to those obtained by PFSDT model or higher order models mentioned as references. The in-plane stresses are also given by such models (Table 6). Values obtained by the DMQPz element are generally, closest to those of Pagano (1970).

$$
\begin{array}{ll}
w=w_{C}\left(100 E_{Y} h^{3} / q_{0} a^{4}\right), & \sigma_{x}^{2}=\sigma_{x}(a / 2, b / 2,0.4 h) h^{2} / q_{0} a^{2}, \\
\sigma_{x}^{1}=\sigma_{x}(a / 2, b / 2, h / 2) h^{2} / q_{0} a^{2}, & \sigma_{y}^{2}=\sigma_{y}(a / 2, b / 2,0.4 h) h^{2} / q_{0} a^{2}, \\
\sigma_{y}^{1}=\sigma_{y}(a / 2, b / 2, h / 2) h^{2} / q_{0} a^{2}, & \tau_{x y}^{1}=\tau_{x y}(0,0, a / 2) h^{2} / q_{0} a^{2}, \\
\tau_{x z}^{1}=\tau_{x z}(0, b / 2,0) h / q_{0} a, & \tau_{y z}^{1}=\tau_{y z}(a / 2,0,0) h q_{0} a, \quad \tau_{x z}=\tau_{x z}(0, b / 2,0.4 h) h / q_{0} a
\end{array}
$$

\section{Conclusion}

Two versions of four-node quadrilateral elements for bending/shear sandwich and multilayer plates are formulated. Based on displacement discrete Mindlin model, in which two discrete Mindlin assumptions are introduced. As a first hypothesis, the kinematics equation of transverse shear strain is assumed as a contour integral and projected on either edge.

It allows the elimination of shear transverse locking without using bubble functions or reduced integration techniques. The second hypothesis requires bending and transverse shear behaviour laws, and two equilibrium plate bending/shear equations. They have a main advantage of local elimination of rotation DOF, initially introduced in the middle elementary edge with quadratic approximation. Thus, the first element baptised DMQP/ml has only three 
Table 6. Normal stresses in simple supported square sandwich (f/c/f) plate, under sinusoidal load.

\begin{tabular}{|c|c|c|c|c|c|c|c|}
\hline & $\begin{array}{l}\mathrm{DMQP} / \mathrm{ml} \\
(8 \times 8)\end{array}$ & $\begin{array}{l}\mathrm{DMQP} / \mathrm{ml} \\
(19 \times 19)\end{array}$ & $\begin{array}{l}\mathrm{DMQPz} \\
(6 \times 6)\end{array}$ & $\begin{array}{l}\text { HOZZT } \\
(8 \times 8)\end{array}$ & $\begin{array}{l}\text { PRHSDT } \\
(8 \times 8)\end{array}$ & $\begin{array}{l}\text { PFSDT } \\
(8 \times 8)\end{array}$ & Pagano \\
\hline \multicolumn{8}{|c|}{$a / h=4$} \\
\hline$\sigma_{x}^{1}$ & .7375 & $.73,125$ & 1.5206 & 1.5158 & 1.4539 & .8385 & 1.556 \\
\hline$\sigma_{x}^{2}$ & .588 & .586 & .2210 & - & .3181 & .6708 & .233 \\
\hline & .0028 & $.00,286$ & .0004 & - & .0012 & .0024 & \\
\hline$\sigma_{v}^{1}$ & .2343 & .235 & .2494 & .2495 & .2522 & .1565 & .2595 \\
\hline \multirow[t]{2}{*}{$\sigma_{y}^{2}$} & .1875 & .188 & .1621 & - & .1631 & .1252 & - \\
\hline & .008 & .008 & .0066 & - & .0069 & .0053 & - \\
\hline \multicolumn{8}{|c|}{$a / h=10$} \\
\hline \multicolumn{2}{|c|}{$\sigma_{r}^{1} 1}$. & 1.02 & 1.1568 & 1.1438 & 1.1453 & 1.0475 & 1.152 \\
\hline \multirow[t]{2}{*}{$\sigma_{x}^{2}$} & .796 & .793 & .6315 & - & .6193 & .8380 & .629 \\
\hline & $.00,215$ & $.00,215$ & $.00,101$ & - & .0018 & .0020 & - \\
\hline$\sigma_{y}^{1}$ & .107 & .107 & .1088 & .1082 & .1101 & .0806 & .1099 \\
\hline \multirow[t]{2}{*}{$\sigma_{y}^{2}$} & .0855 & .0855 & .0823 & - & .0832 & .0645 & - \\
\hline & $.00,364$ & $.00,364$ & $.00,305$ & - & .0035 & .0027 & - \\
\hline
\end{tabular}

degrees of freedom; it presents a good global behaviour and achieves advantages compared to those found in literature. However, in order to fulfil some requirements regarding their local responses, a zigzag form inspired from the Murakami-Reisser-Carrera approach is incorporated in the DDM model. As a result of additional terms, which are displacement type, a new form is formulated without having recourse to the correction technique. The comparison with those based on either higher or improved first-order shear deformation theory shows a further improvement of the DMQP model and yields to obvious accuracy of in-plane and shear stresses.

\section{References}

Ambartsumian, S.A. (1969). Theory of anisotropic plates: Strength, Stability and Vibrations. Translated from Russian by T. Cheron and edited by J. E. Ashton, Technomic.

Ayad, R. (2002). Contribution à la Modélisation numérique pour l'analyse des solides et des structures, et pour la mise en forme des fluides non newtoniens. Application à des matériaux d'emballage (HDR Thesis, University of Reims Champagne Ardenne).

Ayad, R., Dhatt, G., \& Batoz, J.L. (1998). A new hybrid-mixed variational approach for Reissner-Mindlin plates. The MiSP model. International Journal for Numerical Methods in Engineering, 42(7), $1149-1179$.

Ayad, R., \& Rigolot, A. (2002). An improved four-node hybrid-mixed element based upon Mindlin's plate theory. International Journal for Numerical Methods in Engineering, 55(6), 705-731.

Ayad, R., Talbi, N., \& Ghomari, T. (2009). Modified discrete Mindlin hypothesises for laminated composite structures. Composites Science and Technology, 69(1), 125-128. doi: 10.1016/j.compscitech.2007.10.038

Batoz, J.-L., \& Tahar, M.B. (1982). Evaluation of a new quadrilateral thin plate bending element. International Journal for Numerical Methods in Engineering, 18(11), 1655-1677. doi: 10.1002/ nme. 1620181106

Carrera, E. (1996). $C^{0}$ Reissner-Mindlin multilayred plate elements including zig-zag and interlaminar stress continuity. International Journal for Numerical Methods in Engineering, 39(11), 1797-1820.

Carrera, E. (2002). Theories and finite elements for multilayered, anisotropic, composite plates and shells. Archives of Computational Methods in Engineering, 9(2), 87-140. doi: 10.1007/bf02736649 
Carrera, E., \& Demasi, L. (2002). Classical and advanced multilayered plate elements based upon PVD and RMVT. Part 1: Derivation of finite element matrices. International Journal for Numerical Methods in Engineering, 55(2), 191-231. doi: 10.1002/nme.492

Di-Sciuva, M. (1992). Multilayered anisotropic plate models with continuous interlaminar stresses. Composite Structures, 22(3), 149-167. doi: 10.1016/0263-8223(92)90003-u

Engblom, J.J., \& Ochoa, O.O. (1985). Through-the-thickness stress predictions for laminated plates of advanced composite materials. International Journal for Numerical Methods in Engineering, 21(10), 1759-1776. doi: 10.1002/nme.1620211003

Fares, M.E., \& Elmarghany, M.K. (2008). A refined zigzag nonlinear first-order shear deformation theory of composite laminated plates. Composite Structures, 82(1), 71-83. doi: 10.1016/j.compstruct.2006.12.007

Katili, I. (1993). A new discrete Kirchhoff-Mindlin element based on Mindlin-Reissner plate theory and assumed shear strain fields - part II: An extended DKQ element for thick-plate bending analysis. International Journal for Numerical Methods in Engineering, 36(11), 1885-1908. doi: 10.1002/ nme. 1620361107

Lardeur, P. (1990). Développement et évaluation de deux nouveaux éléments finis de plaques et coques composites avec influence du cisaillement transversal $(\mathrm{PhD}$ Thesis, University of Technology of Compiègne, France).

Lekhnitskii, S.G. (1935). Strength calculation of composite beams, Vestnik inzhen i tekhnikov.

Mindlin, R. (1951). Influence of rotatory inertia and shear on flexural motions of isotropic, elastic plates. Journal of Applied Mechanics, 18(1), 31-38.

Murakami, H. (1986). Laminated composite plate theory with improved in-plane responses. Journal of Applied Mechanics, 53, 661-666.

Pagano, N.J. (1970). Exact solutions for rectangular bidirectional composites and sandwich plates. Journal of Composite Materials, 1(4), 257. doi: 10.1016/0010-4361(70)90076-5

Rath, B.K., \& Das, Y.C. (1973). Vibration of layered shells. Journal of Sound and Vibration, 28(4), 737-757. doi: 10.1016/s0022-460x(73)80146-4

Reddy, J.N. (1984). Simple higher-order theory for laminated composite plates. Journal of Applied Mechanics, Transactions ASME, 51(4), 745-752.

Reissner, E. (1943). The effect of transverse shear deformation on the bending of elasticplates. Journal of applied Mechanics, 12, A69-A77.

Sakami S. (2008). Modélisation numérique des structures composites multicouches à l'aide d'une approche discrète au sens de Mindlin. Le modèle DDM (Displacement Discrete Mindlin) (Doctorat Thesis, University of Reims Champagne Ardenne).

Tafla, A., Ayad, R., \& Sedira, L. (2010). A Mindlin multilayered hybrid-mixed approach for laminated and sandwich structures without shear correction factors. Européenne de Mécanique Numérique [European Journal of Computational Mechanics/Revue], 19(8), 725-742. doi: 10.3166/ejcm.19.725-742

Topdar, P., Sheikh, A.H., \& Dhang, N. (2003). Finite element analysis of composite and sandwich plates using a continuous inter-laminar shear stress model. Journal of Sandwich Structures and Materials, 5(3), 207-231. doi: 10.1177/10996362030 05003001.

Whitney, J.M. (1969). The effect of transverse shear deformation on the bending of laminated plates. Journal of Composite Materials, 3(3), 534-547. doi: 10.1177/002199836900300316 